\title{
Log Message Anomaly Detection WITH OVERSAMPLING
}

\author{
Amir Farzad and T. Aaron Gulliver \\ Department of Electrical and Computer Engineering, \\ University of Victoria, PO Box 1700, STN CSC, Victoria, BC Canada V8W 2Y2
}

\begin{abstract}
Imbalanced data is a significant challenge in classification with machine learning algorithms. This is particularly important with log message data as negative logs are sparse so this data is typically imbalanced. In this paper, a model to generate text log messages is proposed which employs a SeqGAN network. An Autoencoder is used for feature extraction and anomaly detection is done using a GRU network. The proposed model is evaluated with three imbalanced log data sets, namely BGL, OpenStack, and Thunderbird. Results are presented which show that appropriate oversampling and data balancing improves anomaly detection accuracy.
\end{abstract}

\section{KEYWORDS}

Deep Learning, Oversampling, Log messages, Anomalydetection, Classification

\section{INTRODUCTION}

Log messages are commonly used in software systems such as cloud servers to record events. These unstructured text messages are typically imbalanced because most logs indicate that the system is working properly and only a small portion indicates a significant problem. Data distribution with a very unequal number of samples for each label is called imbalanced. The problem of imbalanced data has been considered in tasks such as text mining [1], face recognition [2], and software defect prediction [3].

The imbalanced nature of log messages is one of the challenges for classification using deep learning. In binary classification, there are only two labels, and with imbalanced data, most are normal (major) logs. The small number of abnormal (minor) logs makes classification difficult and can lead to poor accuracy with deep learning algorithms. This is because the normal logs dominate the abnormal logs. Oversampling and undersampling are two methods that can be used to address this problem. In undersampling, the major label samples are reduced so the number of minor and major label samples is similar. A serious drawback of undersampling is loss of information [4]. In oversampling, the number of minor label samples is increased so it is similar to the number of major label samples.

Random oversampling and the Synthetic Minority Oversampling Technique (SMOTE) [5] are two well-known oversampling methods. Random oversampling increases the minor samples by randomly repeating some minor samples. SMOTE creates synthetic minority samples to increase their number. The performance of SMOTE is generally better than with random oversampling [5]. However, random oversampling and SMOTE provide poor performance when used to oversample log messages [6]. A generative adversarial network (GAN) [7] is effective in 
International Journal of Artificial Intelligence and Applications (IJAIA), Vol.11, No.4, July 2020

generating images that are similar to actual images [8]. GANs can produce more abstract and varied data than other algorithms [9].

In this paper, a model is proposed to deal with imbalanced log data by oversampling text log messages using a Sequence Generative Adversarial Network (SeqGAN) [10]. The resulting data is then used for anomaly detection and classification with Autoencoder [11] and Gated Recurrent Unit (GRU) [12] networks. An Autoencoder is a feed-forward network that is effective in extracting important information from data. Autoencoders have been applied to many tasks such as probabilistic and generative modeling [13] and representation learning [14]. A GRU is a Recurrent Neural Network (RNN) that has been used for tasks such as sentiment analysis [15] and speech recognition [16]. The proposed model is evaluated using three labeled log message data sets, namely BlueGene/L (BGL), OpenStack, and Thunderbird. Results are presented which show that the proposed model with oversampling provides better results than without oversampling.

The main contributions of this paper are as follows.

1. A new model is proposed for log message oversampling for anomaly detection and classification using SeqGAN, Autoencoder, and GRU networks.

2. Model results with and without log message oversampling are compared.

3. Three well-known data sets are used to evaluate the model.

The rest of this paper is organized as follows. In Section 2 the Autoencoder, GRU, and SeqGAN architectures are given and the proposed model is described. Section 3 presents the experimental results and discussion, while Section 4 provides some conclusions.

\section{SySTEM MODEL}

In this section, the Autoencoder, GRU, and SeqGAN architectures are given along with the proposed network model.

\subsection{Autoencoder Architecture}

An Autoencoder is a feed-forward multi-layer neural network with the same number of input and output neurons. It is used to learn an efficient representation of data while minimizing the error. An Autoencoder with more than one hidden layer is called a deep Autoencoder [17]. A reduced dimension data representation is produced using encoder and decoder hidden layers in the Autoencoder architecture. Backpropagation is used for training to reduce the loss based on a loss function. Figure 1 shows the Autoencoder architecture with an input layer, a hidden layer, and an output layer.

\subsection{GRU Architecture}

A Gated Recurrent Unit (GRU) is a type of RNN network which is a modified version of an Long Short-Term Memory (LSTM) network [18]. It has a reset gate and an update gate. The reset gate determines how much information in a block should be forgotten and is given by

$$
r_{t}=\sigma\left(W_{r} x_{t}+U_{r} h_{t-1}+b_{r}\right),
$$


International Journal of Artificial Intelligence and Applications (IJAIA), Vol.11, No.4, July 2020

where $b_{r}$ is a bias vector, $\sigma$ is the sigmoid activation function, and $W_{r}$ and $U_{r}$ are weight matrices. The update gate decides how much information should be updated and can be expressed as

$$
z_{t}=\sigma\left(W_{z} x_{t}+U_{z} h_{t-1}+b_{z}\right)
$$

where $W_{z}$ and $U_{z}$ are weight matrices and $b_{z}$ is a bias vector. The block output at time $t$ is

$\left.h_{\mathrm{t}}=z_{\mathrm{t}} \times h_{\mathrm{t}-1}+\left(1-z_{\mathrm{t}}\right) \times \tanh \left(W_{h} x_{\mathrm{t}}\right)+U_{h}\left(r_{\mathrm{t}} \times h_{\mathrm{t}-1}\right)+b_{h}\right)$,

where $b_{h}$ is a bias vector, and $W_{h}$ and $U_{h}$ are weight matrices. A GRU block is shown in Figure 2 .

\subsection{SEQGAN Architecture}

A SeqGAN consists of a Generator $(G)$ and Discriminator $(D)$. The Discriminator is trained to discriminate between real data (sentences) and generated sentences. The Generator is trained using the Discriminator using a reward function with policy gradient [19]. The reward for a sentence is used to regulate the Generator via reinforcement learning. Generator $G_{\theta}$ is trained with a real data set to produce a sentence

$$
Y_{1: T}=\left\{y_{1}, \ldots, y_{t}, \ldots, y_{T}\right\}, y_{t} \in \mathrm{Y}
$$

where $\mathrm{Y}$ is the vocabulary of candidate words. This should produce a sentence that is close to real data. This is a reinforcement learning problem which considers $G_{\theta}$ to produce an action $a$ (next word $y_{t}$ ) given the state $s$ (previously generated words $Y_{1: t-1}$ ). SeqGAN trains the Discriminator $D_{\phi}$ as well as $G_{\theta} . D_{\phi}$ is trained to discriminate between real data and data generated by $G_{\theta}$. Words are generated by $G_{\theta}$ each time step but $D_{\phi}$ only computes rewards for full sentences. Hence, the rewards for intermediate states are estimated using Monte Carlo (MC) search and are given by

$$
\begin{gathered}
Q_{D_{\phi}}^{G_{\theta}}=\left(s=Y_{1: t-1}, a=y_{t}\right)= \\
\left\{\begin{array}{l}
\frac{1}{N} \sum_{n=1}^{N} D_{\phi}\left(Y_{1: T}^{n}\right), Y_{1: T}^{n} \in M C\left(Y_{1: t} ; N\right) \quad \text { if }: t<T, \\
D_{\phi}\left(Y_{1: t}\right) \quad \text { if }: t=T,
\end{array}\right.
\end{gathered}
$$

where $Q_{D_{\phi}}^{G_{\theta}}$ is the action-value function which is the expected reward from the Discriminator, $T$ is the sentence length and $N$ is the number of sentences in the MC search, $Y_{1: T}^{n}$ is the $n$th sentence in the MC search, and $D_{\phi}\left(Y_{1: T}^{n}\right)$ is the probability of the $n$th sentence denoted real by the Discriminator. After the reward is computed, $G_{\theta}$ is updated via the policy gradient which is the gradient of the objective function and is given by 


$$
\begin{gathered}
\nabla_{\theta} J(\theta)=\frac{1}{T} \sum_{t=1}^{T} \sum_{y_{t} \in \mathrm{Y}} \nabla_{\theta} G_{\theta}\left(y_{t} \mid Y_{1: t-1}\right) Q_{D_{\phi}}^{G_{\theta}}\left(Y_{1: t-1}, y_{t}\right) \\
=\frac{1}{T} \sum_{t=1}^{T} \mathrm{E}_{y_{t}=G_{\theta}\left(y_{t} \mid Y_{1: t-1}\right)}\left[\nabla_{\theta} \log G_{\theta}\left(y_{t} \mid Y_{1: t-1}\right) Q_{D_{\phi}}^{G_{\theta}}\left(Y_{1: t-1}, y_{t}\right)\right], \\
\theta \leftarrow \theta+\alpha \nabla_{\theta} J(\theta),
\end{gathered}
$$

where $\alpha$ is the learning rate. SeqGAN updates the Discriminator and Generator until the stopping criteria are satisfied. An LSTM, GRU, or other RNN network for the Generator and a Convolutional Neural Network $(\mathrm{CNN})$ for the Discriminator have been shown to provide good results for classification tasks [10].

The SeqGAN architecture is shown in Figure 3. The orange circles denote words in real sentences and the blue circles denote words in generated sentences. First, the Generator is pretrained with real data using the cross-entropy loss function which minimizes the negative loglikelihood. Then it is used to generate data and the Discriminator is pretrained with both generated and real data. The MC search parameters $(\beta)$ are set to be the same as the Generator parameters $(\theta)$. As shown on the right, an MC search is used to compute the reward for an intermediate state. This search generates $N$ complete sentences from the current state. A reward is computed for each sentence and averaged as the intermediate reward except in the last time step where the reward is obtained from the Discriminator. The input of each time step is the output of the previous step and the next word is obtained via a multinomial distribution over the softmax of the GRU output. Then the Generator is trained using the policy gradient. Finally, the updated Generator is used to generate data and the Discriminator is trained with both the generated and real data. The SeqGAN algorithm is given in Algorithm 1.

\section{Algorithm 1 The SeqGAN algorithm}

1: Pretrain Generator $G_{\theta}$ with real data

2: $\beta \leftarrow \theta$

3: Pretrain Discriminator $D_{\phi}$ using the data generated by the Generator and real data

4: repeat

5: $\quad$ for $\mathrm{g}$-steps do

6: $\quad$ Generate a sentence $Y_{1: T}=\left\{y_{1}, \ldots, y_{T}\right\} \sim G_{\theta}$

7: $\quad$ for $t$ in $1: T$ do

8: $\quad$ Compute the rewards using (4)

9: $\quad$ Update the Generator parameters with the policy gradient using (6)

10: for d-steps do

11: Use the Generator to generate data

12: $\quad$ Train the Discriminator with real and generated data

13: $\beta \leftarrow \theta$

14: until stopping criteria are satisfied

\subsection{Proposed Model}

The proposed model has three steps. The first is generating log messages using SeqGAN for oversampling. The log messages are divided into two data sets, positive labeled data (normal) and 
International Journal of Artificial Intelligence and Applications (IJAIA), Vol.11, No.4, July 2020

negative labeled data (abnormal). Additional negative labeled data is generated using the negative labeled data set. The initial negative data set is split into sets (the OpenStack data set is split into two sets while the BGL and Thunderbird data sets are split into seven sets), and each set is input to the SeqGAN separately. This ensures better convergence and provides different negative log messages. Further, this improves the speed which is important with data generation. A CNN is used in the SeqGAN for the discriminator and a GRU as the generator. The GRU has one hidden layer of size 30 with the ADAM optimizer and the batch size is 128 . The generated negative log messages are concatenated with the original negative data and similar messages are removed. The resulting data set is balanced with similar numbers of positive and negative data.

The second step is the Autoencoder which has two networks (positive and negative) with three hidden layers (two encoder layers and one decoder layer). The encoder layers have 400 (with L1 regularizer) and 200 neurons and the decoder layer has 200 neurons. The output layer has 40 neurons which is the same size as the input layer. The positive labeled data is fed into the positive Autoencoder. Note that this network is trained with just positive labeled data. The maximum number of epochs is 100 and the batch size is 128 . Dropout with probability 0.8 and early stopping are used to prevent overfitting. Categorical cross-entropy loss with the ADAM optimizer is used for training. The network output is labeled as positive. The negative labeled data which has been oversampled is fed into the negative Autoencoder and the network output is labeled as negative. The two sets of labeled data are then concatenated, duplicates are removed and Gaussian noise with zero mean and variance 0.1 is added to avoid overfitting [20].

The final step is the GRU network for anomaly detection and classification. First, the concatenated data set is divided into training and testing sets with 5\% for training and $95 \%$ for testing, and these sets are shuffled. The training set is then divided into two sets with 5\% for training and $95 \%$ for validation. The data is fed into the GRU with hidden layer size 100 and is classified using softmax activation. 10-fold cross-validation is used in training with a maximum of 100 epochs and a batch size of 128 . Dropout with probability 0.8 and early stopping are used to prevent overfitting. Categorical cross-entropy loss with the ADAM optimizer is used for training. The proposed model is shown in Figure 4.

\section{RESUlts}

In this section, the proposed model is evaluated with and without SeqGAN oversampling using the BGL, OpenStack, and Thunderbird data sets. Four criteria are used to evaluate the performance, namely accuracy, precision, recall, and F-measure. Accuracy is the fraction of the input data that is correctly predicted and is given by

$$
A=\frac{T_{p}+T_{n}}{T_{p}+T_{n}+F_{p}+F_{n}},
$$

where $T_{p}$ is the number of positive instances predicted by the model to be positive, $T_{n}$ is the number of negative instances predicted to be negative, $F_{p}$ is the number of negative instances predicted to be positive, and $F_{n}$ is the number of positive instances predicted to be negative. Precision is given by

$$
P=\frac{T_{p}}{T_{p}+F_{p}},
$$

and recall is 


$$
R=\frac{T_{p}}{T_{p}+F_{n}} .
$$

The F-measure is the harmonic mean of recall and precision which can be expressed as

$$
F=\frac{2 \times P \times R}{P+R} .
$$

All experiments were conducted on the Compute Canada Cedar cluster with 24 CPU cores, 125 GB memory, and four P100 GPUs with Python in Keras and Tensorflow. The hyperparameters of the proposed model were not tuned so the default values were used for all data sets. For each data set, the average training accuracy, average validation accuracy, average training loss, testing accuracy, precision, recall, and F-measure were obtained. Tables 1 and 2 give the results for the BGL, OpenStack, and Thunderbird data sets without and with SeqGAN oversampling, respectively.

\subsection{BGL}

The BlueGene/L (BGL) data set consists of 4,399,503 positive log messages and 348,460 negative log messages (without oversampling). From this data set, 11,869 logs are used for training, 225,529 for validation, and the remaining 4,510,565 for testing with approximately $95 \%$ positive and $5 \%$ negative messages in each group. Without oversampling, the average training accuracy is $97.8 \%$ and average validation accuracy is $98.6 \%$ with standard deviations of 0.02 and 0.01 , respectively, in 10-fold cross-validation. The average training loss is 0.07 with a standard deviation of 0.01 . The testing accuracy is $99.3 \%$ with a precision of $98.9 \%$ for negative logs and 99.3\% for positive logs, and recall of $91.6 \%$ and $99.9 \%$ for negative and positive logs, respectively. The F-measure is $95.1 \%$ and $99.6 \%$ for negative and positive logs, respectively.

Oversampling of the negative log messages with SeqGAN increased the number in the data set to $4,137,516$ so the numbers of positive and negative log messages are similar. From this data set, $21,342 \operatorname{logs}$ are used for training, 405,508 for validation, and the remaining 8,110,169 for testing with similar numbers of positive and negative log messages in each group. The average training accuracy is $98.3 \%$ and average validation accuracy is $99.3 \%$ with a standard deviation of 0.01 in 10 -fold cross-validation. The average training loss is 0.05 with a standard deviation of 0.01 . The testing accuracy is $99.6 \%$ with a precision of $99.8 \%$ for negative logs and $99.4 \%$ for positive logs, and recall of $99.3 \%$ and $99.8 \%$ for negative and positive logs, respectively. The F-measure is $99.6 \%$ for both negative and positive logs. The precision, recall, and F-measure with oversampling for negative logs are better than the values of $99 \%, 75 \%$, and $85 \%$, respectively, with SVM supervised learning and $83 \%, 99 \%$, and $91 \%$, respectively, with unsupervised learning [21].

\subsection{Open Stack}

The OpenStack data set without oversampling consists of 137,074 positive log messages and 18,434 negative log messages. From this data set, 6,608 logs are used for training, 1,167 for validation, and the remaining 147,733 for testing with approximately $95 \%$ positive and $5 \%$ negative messages in each group. Without oversampling, the average training accuracy is $98.4 \%$ and average validation accuracy is $97.2 \%$ with a standard deviation of 0.01 in 10-fold crossvalidation. The average training loss is 0.05 with a standard deviation of 0.01 . The testing 
International Journal of Artificial Intelligence and Applications (IJAIA), Vol.11, No.4, July 2020

accuracy is $98.3 \%$ with a precision of $97.9 \%$ for negative logs and $98.3 \%$ for positive logs, and recall of $87.1 \%$ and $99.8 \%$ for negative and positive logs, respectively. The F-measure is $92.2 \%$ and $99.0 \%$ for negative and positive logs, respectively.

Oversampling of the negative log messages with SeqGAN increased the number in the data set to 154,202 . From this data set, $12,378 \operatorname{logs}$ are used for training, 2,185 for validation, and the remaining 276,713 for testing with similar numbers of positive and negative log messages in each group. With oversampling, the average training accuracy is $98.0 \%$ and average validation accuracy is $98.7 \%$ with a standard deviation of 0.01 in 10 -fold cross-validation. The average training loss is 0.06 with a standard deviation of 0.01 . The testing accuracy is $98.9 \%$ with a precision of $99.6 \%$ for negative logs and $98.2 \%$ for positive logs, and recall of $98.4 \%$ and $99.5 \%$ for the negative and positive logs, respectively. The F-measure is $99.0 \%$ and $98.8 \%$ for negative and positive logs, respectively. The precision, recall, and F-measure with oversampling for negative logs are better than the $94 \%, 99 \%$, and $97 \%$ obtained with the Deeplog network [22].

\subsection{Thunderbird}

From the Thunderbird data set, 3,000,000 positive log messages and 300,000 negative log messages (without oversampling) were selected. From this data set, 8,250 logs are used for training, 156,750 for validation, and the remaining 3,135,000 for testing with approximately $95 \%$ positive and $5 \%$ negative messages in each group. Without oversampling, the average training accuracy is $98.7 \%$ and average validation accuracy is $98.8 \%$ with a standard deviation of 0.01 in 10 -fold cross-validation. The average training loss is 0.04 with a standard deviation of 0.02 . The testing accuracy is $98.5 \%$ with a precision of $93.2 \%$ for negative logs and $99.1 \%$ for positive logs, and recall of $90.6 \%$ and $99.3 \%$ for negative and positive logs, respectively. The F-measure is $91.9 \%$ and $99.2 \%$ for negative and positive logs, respectively.

Oversampling of the negative log messages with SeqGAN increased the number in the data set to $3,000,000$ so the numbers of positive and negative log messages are the same. From this data set, $15,000 \operatorname{logs}$ are used for training, 285,000 for validation, and the remaining 5,700,000 for testing with similar numbers of positive and negative log messages in each group. The average training accuracy is $99.1 \%$ and average validation accuracy is $99.3 \%$ with standard deviations of 0.01 and 0.02 , respectively, in 10-fold cross-validation. The average training loss is 0.04 with a standard deviation of 0.01 . The testing accuracy is $99.6 \%$ with a precision of $99.5 \%$ for negative logs and $99.7 \%$ for positive logs, and recall of $99.7 \%$ and $99.5 \%$ for negative and positive logs, respectively. The F-measure is $99.6 \%$ for both negative and positive logs. The precision, recall, and F-measure with oversampling for negative logs are better than the 96\%, 96\%, and 96\%, respectively, with the Improved K-Nearest Neighbor algorithm [23].

\subsection{Discussion}

The results obtained show that the oversampling with SeqGAN provided good results for the BGL, OpenStack, and Thunderbird data sets. It is evident that oversampling significantly improved the model accuracy for negative log messages. For the BGL data set, the precision, recall and F-measure after oversampling increased from $98.9 \%$ to $99.8 \%, 91.6 \%$ to $99.3 \%$ and $95.1 \%$ to $99.6 \%$ which are $0.9 \%, 7.7 \%$ and $4.5 \%$ higher, respectively. For the OpenStack data set, the precision, recall and F-measure after oversampling increased from $97.9 \%$ to $99.6 \%$, $87.1 \%$ to $98.4 \%$ and $92.2 \%$ to $99.0 \%$ which are $1.7 \%, 11.3 \%$ and $6.8 \%$ higher, respectively. For the Thunderbird data set, the precision, recall, and F-measure after oversampling increased from $93.2 \%$ to $99.5 \%, 90.6 \%$ to $99.7 \%$, and $91.9 \%$ to $99.6 \%$ which are $6.3 \%, 9.1 \%$, and $7.7 \%$ higher, respectively. This indicates that data balancing should be considered with deep learning algorithms to improve the performance. This is particularly important when the number of minor 
International Journal of Artificial Intelligence and Applications (IJAIA), Vol.11, No.4, July 2020

label samples is very small. The proposed model was evaluated with three data sets for anomaly detection and classification with only a small portion (less than 1\%) used for training. This is very significant as deep learning algorithms typically require significant amounts of data for training [24]. Note that good results were obtained even though the hyperparameters were not tuned.

The most important step in the proposed model is oversampling with a SeqGAN network. These networks have been shown to provide good results in generating text such as poems [25]. The concept of generating data is similar to that for oversampling. It was interesting that duplication in the oversampled log data was not high (less than 5\%). As a consequence, after removing duplicates a significant amount of data was available for anomaly detection and classification. Feature extraction using an Autoencoder is also important. The output from the Autoencoder is suitable for use with an RNN-based algorithm such as a GRU for anomaly detection and classification. This was confirmed by the results obtained which show that the proposed model provides excellent performance even when the data is imbalanced.

\section{Conclusion}

In this paper, a model was proposed to address the problem of imbalanced log message data. In the first step, the negative logs were oversampled with a SeqGAN network so that the numbers of positive and negative logs are similar. The resulting labeled logs were then fed into an Autoencoder to extract features and information from the text data. Finally, a GRU network was used for anomaly detection and classification. The proposed model was evaluated using three log message data sets, namely BGL, OpenStack, and Thunderbird. Results were presented which show that oversampling can improve detection and classification accuracy. In the future, other text-based GAN networks such as TextGAN and MaliGAN can be used for oversampling.

Table 1. Results without oversampling for the BGL, OpenStack, and Thunderbird data sets (numbers in parenthesis are standard deviation). Positive labels are denoted by 1 and negative labels by 0 .

\begin{tabular}{|c|c|c|c|c|c|c|c|c|}
\hline Data set & $\begin{array}{l}\text { Average } \\
\text { Training } \\
\text { Accuracy }\end{array}$ & $\begin{array}{l}\text { Average } \\
\text { Validation } \\
\text { Accuracy }\end{array}$ & $\begin{array}{l}\text { Average } \\
\text { Training } \\
\text { Loss }\end{array}$ & $\begin{array}{l}\text { Testing } \\
\text { Accuracy }\end{array}$ & Label & Precision & Recall & F-measure \\
\hline \multirow[t]{2}{*}{ BGL } & $97.8 \%$ & $98.6 \%$ & 0.07 & $99.3 \%$ & 0 & $98.9 \%$ & $91.6 \%$ & $95.1 \%$ \\
\hline & $(0.02)$ & $(0.01)$ & $(0.01)$ & & 1 & $99.3 \%$ & $99.9 \%$ & $99.6 \%$ \\
\hline \multirow[t]{2}{*}{ OpenStack } & $98.4 \%$ & $97.2 \%$ & 0.05 & $98.3 \%$ & 0 & $97.9 \%$ & $87.1 \%$ & $92.2 \%$ \\
\hline & $(0.01)$ & $(0.01)$ & $(0.01)$ & & 1 & $98.3 \%$ & $99.8 \%$ & $99.0 \%$ \\
\hline \multirow{3}{*}{ Thunderbird } & $98.7 \%$ & $98.8 \%$ & 0.04 & & 0 & $93.2 \%$ & $90.6 \%$ & $91.9 \%$ \\
\hline & & & & $98.5 \%$ & & & & \\
\hline & $(0.01)$ & $(0.01)$ & $(0.02)$ & & 1 & $99.1 \%$ & $99.3 \%$ & $99.2 \%$ \\
\hline
\end{tabular}


International Journal of Artificial Intelligence and Applications (IJAIA), Vol.11, No.4, July 2020

Table 2. Results with oversampling using SeqGAN for the BGL, OpenStack, and Thunderbird data sets (numbers in parenthesis are standard deviation). Positive labels are denoted by 1 and negative labels by 0 .

\begin{tabular}{|c|c|c|c|c|c|c|c|c|}
\hline Data set & $\begin{array}{l}\text { Average } \\
\text { Training } \\
\text { Accuracy }\end{array}$ & $\begin{array}{l}\text { Average } \\
\text { Validation } \\
\text { Accuracy }\end{array}$ & $\begin{array}{c}\text { Average } \\
\text { Training } \\
\text { Loss }\end{array}$ & $\begin{array}{l}\text { Testing } \\
\text { Accuracy }\end{array}$ & Label & Precision & Recall & F-measure \\
\hline BGL & $\begin{array}{l}98.3 \% \\
(0.01)\end{array}$ & $\begin{array}{l}99.3 \% \\
(0.01)\end{array}$ & $\begin{array}{c}0.05 \\
(0.01)\end{array}$ & $99.6 \%$ & $\begin{array}{l}0 \\
1\end{array}$ & $\begin{array}{l}99.8 \% \\
99.4 \%\end{array}$ & $\begin{array}{l}99.3 \% \\
99.8 \%\end{array}$ & $\begin{array}{l}99.6 \% \\
99.6 \%\end{array}$ \\
\hline OpenStack & $\begin{array}{l}98.0 \% \\
(0.01)\end{array}$ & $\begin{array}{l}98.7 \% \\
(0.01)\end{array}$ & $\begin{array}{c}0.06 \\
(0.01)\end{array}$ & $98.9 \%$ & $\begin{array}{l}0 \\
1\end{array}$ & $\begin{array}{l}99.6 \% \\
98.2 \%\end{array}$ & $\begin{array}{l}98.4 \% \\
99.5 \%\end{array}$ & $\begin{array}{l}99.0 \% \\
98.8 \%\end{array}$ \\
\hline Thunderbird & $\begin{array}{l}99.1 \% \\
(0.01)\end{array}$ & $\begin{array}{l}99.3 \% \\
(0.02)\end{array}$ & $\begin{array}{c}0.04 \\
(0.01)\end{array}$ & $99.6 \%$ & 0 & $99.5 \%$ & $99.7 \%$ & $99.6 \%$ \\
\hline
\end{tabular}

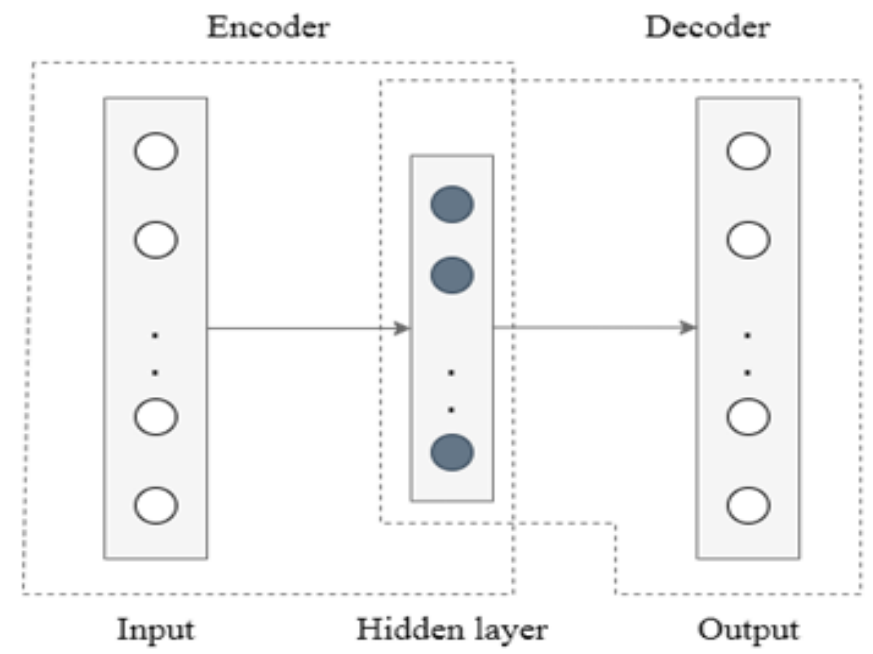

Figure 1. Autoencoder architecture with an input layer, a hidden layer, and an output layer. 
International Journal of Artificial Intelligence and Applications (IJAIA), Vol.11, No.4, July 2020

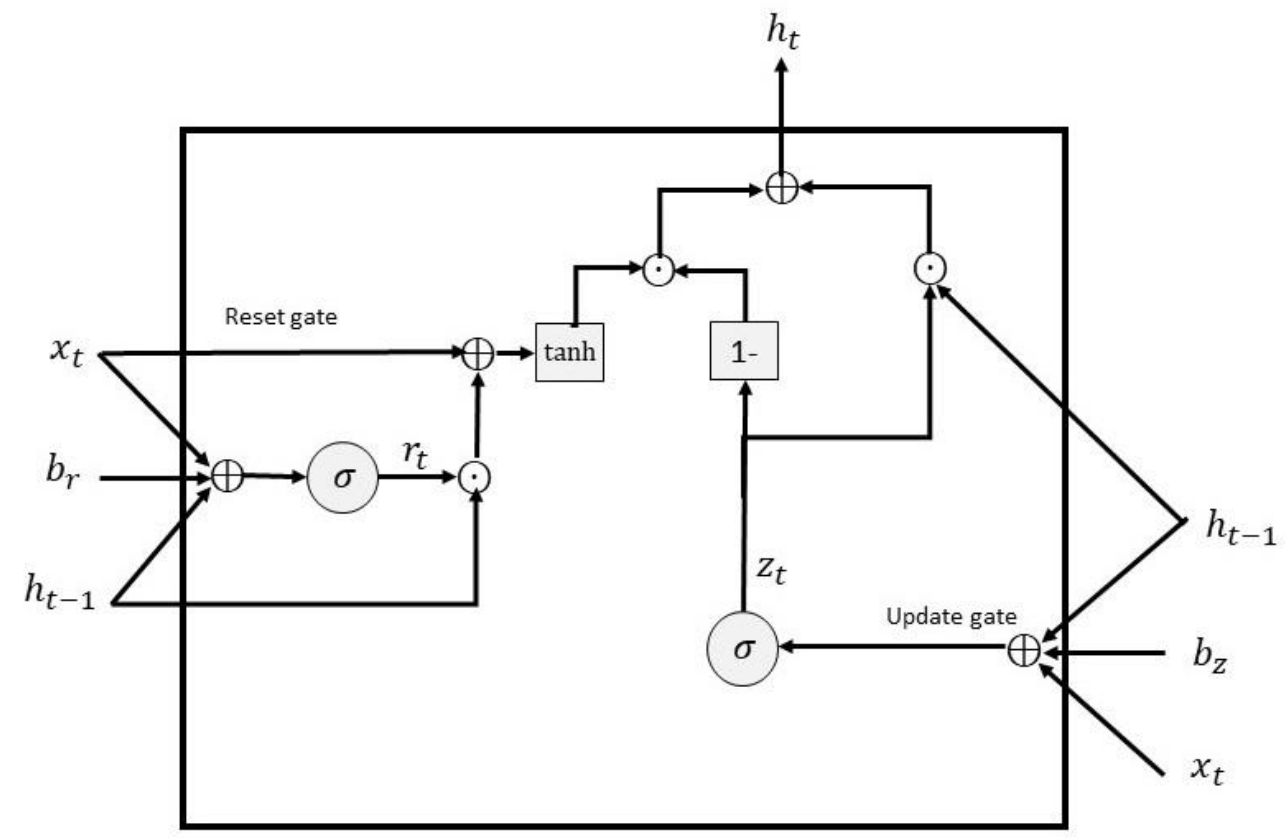

Figure 2. A GRU block with reset gate, update gate, and tangent hyperbolic and sigmoid activation functions.

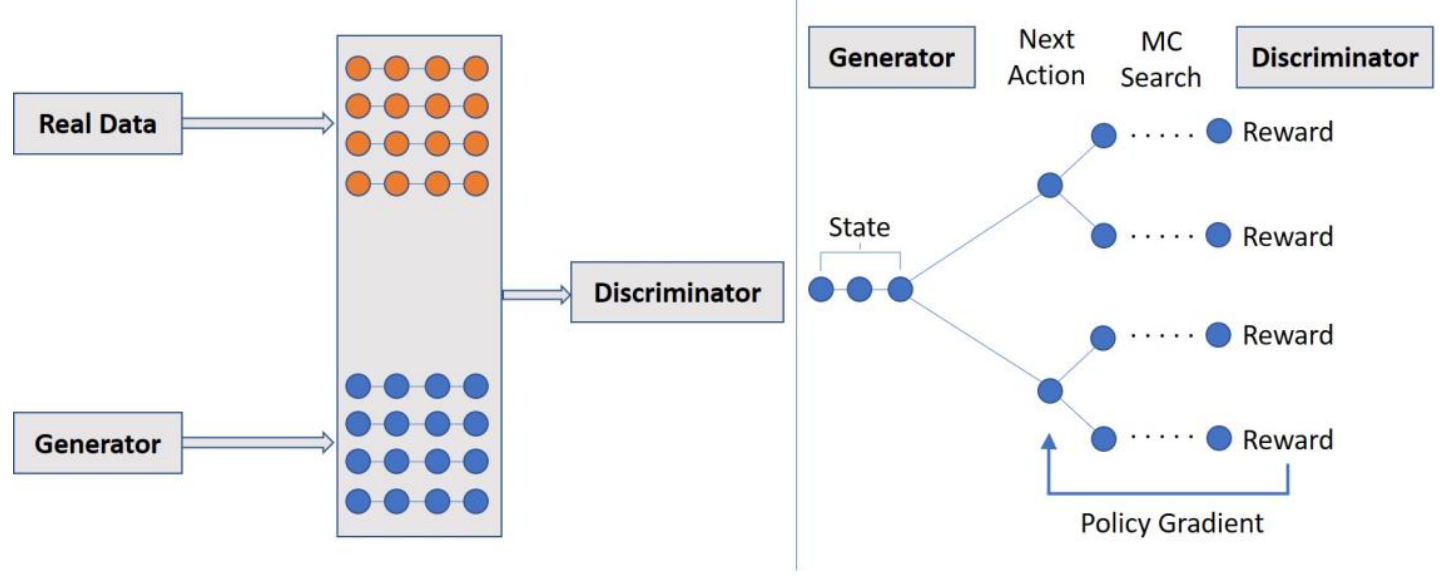

Figure 3. The SeqGAN architecture. The Discriminator on the left is trained with real data and data is generated using the Generator. The Generator on the right is trained using the policy gradient. The reward is computed by the Discriminator and this is used to determine the intermediate action values for the MC search. 
International Journal of Artificial Intelligence and Applications (IJAIA), Vol.11, No.4, July 2020

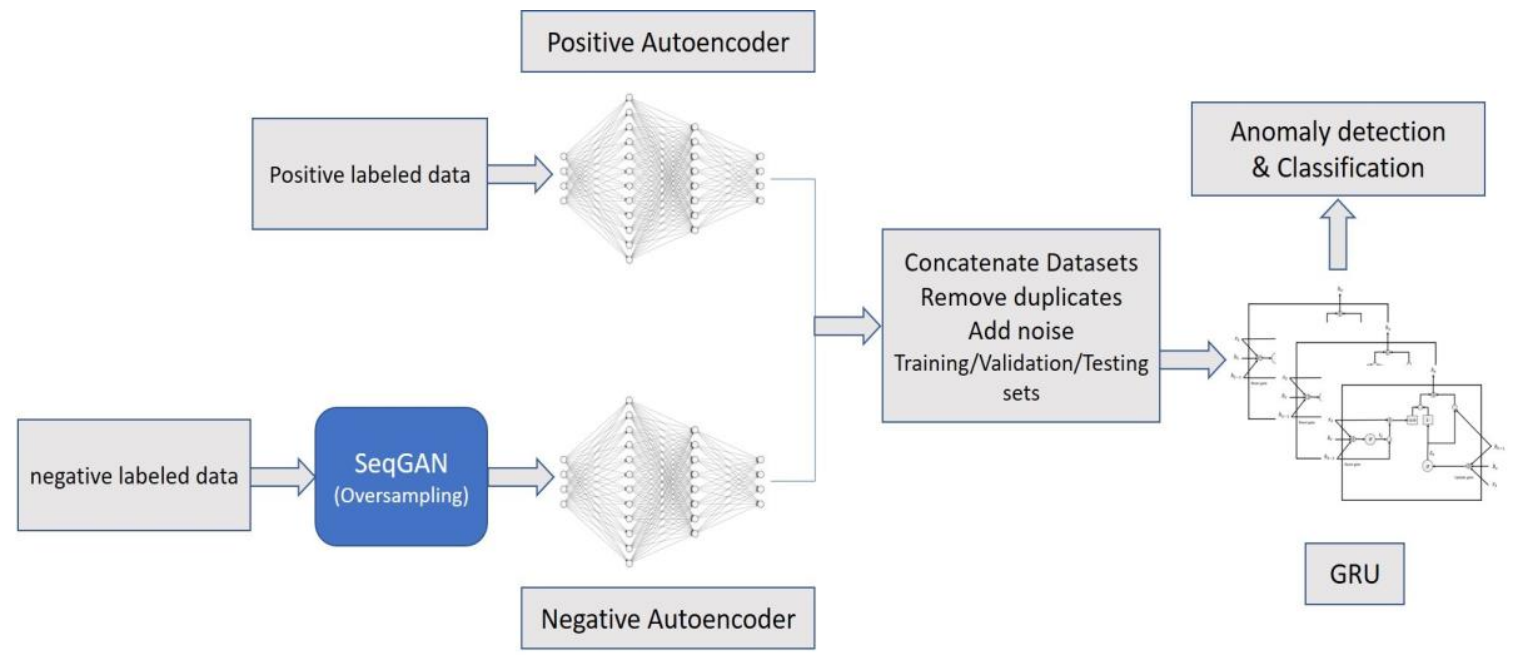

Figure 4. The proposed model architecture with SeqGAN for oversampling log messages, two Autoencoder networks, and a GRU network for anomaly detection and classification.

\section{REFERENCES}

[1] T. Munkhdalai, O.-E. Namsrai and K. H. Ryu, "Self-training in Significance Space of Support Vectors for Imbalanced Biomedical Event Data", BMC Bioinformatics, vol. 16, no. S7, pp. 1-8, 2015.

[2] Y.-H. Liu and Y.-T. Chen, "Total Margin Based Adaptive Fuzzy Support Vector Machines for Multiview Face Recognition", in IEEE International Conference on Systems, Man and Cybernetics, pp. 1704-1711, 2005.

[3] M. J. Siers and M. Z. Islam, "Software Defect Prediction using A Cost Sensitive Decision Forest and Voting, and A Potential Solution to the Class Imbalance Problem", Information Systems, vol. 51, pp. 62-71, 2015.

[4] N. V. Chawla, "Data Mining for Imbalanced Datasets: An Overview", in Data Mining and Knowledge Discovery Handbook, pp. 853-867, Springer, 2005.

[5] N. V. Chawla, K. W. Bowyer, L. O. Hall and W. P. Kegelmeyer, "SMOTE: Synthetic Minority Oversampling Technique", Journal of Artificial Intelligence Research, vol. 16, pp. 321-357, 2002.

[6] B. Krawczyk, "Learning from Imbalanced Data: Open Challenges and Future Directions", Artificial Intelligence, vol. 5, no. 4, pp. 221-232, 2016.

[7] I. Goodfellow, J. Pouget-Abadie, M. Mirza, B. Xu, D. Warde-Farley, S. Ozair, A. Courville and Y. Bengio, "Generative Adversarial Nets", in International Conference on Neural Information Processing Systems, pp. 2672-2680, MIT Press, 2014.

[8] D. Li, Q. Huang, X. He, L. Zhang and M.-T. Sun, "Generating Diverse and Accurate Visual Captions by Comparative Adversarial Learning", arXiv e-prints, arXiv:1804.00861, 2018.

[9] L. Liu, Y. Lu, M. Yang, Q. Qu, J. Zhu and H. Li, "Generative Adversarial Network for Abstractive Text Summarization", arXiv e-prints, p. arXiv:1711.09357, 2017.

[10] L. Yu, W. Zhang, J. Wang and Y. Yu, "SeqGAN: Sequence Generative Adversarial Nets with Policy Gradient", in AAAI Conference on Artificial Intelligence, pp. 2852-2858, 2017.

[11] D. E. Rumelhart and J. L. McClelland, Parallel Distributed Processing: Explorations in the Microstructure of Cognition: Foundations, ch. Learning Internal Representations by Error Propagation, pp. 318-362. MIT Press, 1986.

[12] K. Cho, B. Merriënboer, C. Gulcehre, D. Bahdanau, F. Bougares, H. Schwenk and Y. Bengio, "Learning Phrase Representations Using RNN Encoder-Decoder for Statistical Machine Translation", in Conference on Empirical Methods in Natural Language Processing, pp. 1724-1734, 2014. 
International Journal of Artificial Intelligence and Applications (IJAIA), Vol.11, No.4, July 2020

[13] D. J. Rezende, S. Mohamed and D. Wierstra, "Stochastic Backpropagation and Approximate Inference in Deep Generative Models", in International Conference on Machine Learning, vol. 32, pp. II-1278-II1286, 2014.

[14] I. Higgins, L. Matthey, A. Pal, C. Burgess, X. Glorot, M. Botvinick, S. Mohamed and A. Lerchner, "Beta-VAE: Learning Basic Visual Concepts with a Constrained Variational Framework", in International Conference on Learning Representations, 2017.

[15] M. Kuta, M. Morawiec and J. Kitowski, "Sentiment Analysis with Tree-Structured Gated Recurrent Units", in Text, Speech, and Dialogue, Lecture Notes in Artificial Intelligence, vol. 10415, pp. 74-82, Springer, 2017.

[16] K. Irie, Z. Tüske, T. Alkhouli, R. Schlüter and H. Ney, "LSTM, GRU, Highway and a Bit of Attention: An Empirical Overview for Language Modeling in Speech Recognition", in Interspeech, pp. 35193523, 2016.

[17] Y. LeCun, Y. Bengio and G. Hinton, "Deep Learning”, Nature, vol. 521, no. 7553, pp. 436-444, 2015.

[18] S. Hochreiter and J. Schmidhuber, "Long Short-Term Memory", Neural Computation, vol. 9, no. 8, pp. 1735-1780, 1997.

[19] R. S. Sutton, D. McAllester, S. Singh and Y. Mansour, "Policy Gradient Methods for Reinforcement Learning with Function Approximation", in International Conference on Neural Information Processing Systems, pp. 1057-1063, MIT Press, 1999.

[20] H. Noh, T. You, J. Mun and B. Han, "Regularizing Deep Neural Networks by Noise: Its Interpretation and Optimization", in Advances in Neural Information Processing Systems, (I. Guyon, U. V. Luxburg, S. Bengio, H. Wallach, R. Fergus, S. Vishwanathan, and R. Garnett, eds.), vol. 30, pp. 5109-5118, Curran Associates, 2017.

[21] S. He, J. Zhu, P. He and M. R. Lyu, "Experience Report: System Log Analysis for Anomaly Detection", in IEEE International Symposium on Software Reliability Engineering, pp. 207-218, 2016.

[22] M. Du, F. Li, G. Zheng and V. Srikumar, "DeepLog: Anomaly Detection and Diagnosis from System Logs through Deep Learning", in ACM Conference on Computer and Communications Security, pp. 1285-1298, 2017.

[23] B. Wang, S. Ying, G. Cheng, R. Wang, Z. Yang and B. Dong, "Log-Based Anomaly Detection with the Improved K-Nearest Neighbor", International Journal of Software Engineering and Knowledge Engineering, vol. 30, no. 2, pp. 239-262, 2020.

[24] A. Farzad and T. A. Gulliver, "Oversampling Log Messages Using a Sequence Generative Adversarial Network for Anomaly Detection and Classication", in International Conference on Artificial Intelligence and Machine Learning, vol. 10, no. 5, pp. 163-175, 2020.

[25] X. Wu, M. Klyen, K. Ito and Z. Chen, "Haiku Generation using Deep Neural Networks", in Annual Conference of the Language Processing Society, pp. 1133-1136, 2017. 
International Journal of Artificial Intelligence and Applications (IJAIA), Vol.11, No.4, July 2020

\section{AUTHORS}

Amir Farzad received the Masters degree in computer engineering (minor: artificial intelligence) from Shahrood University of Technology, Shahrood, Iran, in 2016. He is currently a Ph.D. student in the Department of Electrical and Computer Engineering, University of Victoria, Victoria, BC, Canada. His research interests include machine learning, deep learning, and natural language processing.

Thomas Aaron Gulliver received the Ph.D. degree in electrical engineering from the University of Victoria, Victoria, BC, Canada, in 1989. From 1989 to 1991, he was a Defence Scientist with the Defence Research Establishment Ottawa, Ottawa, ON, Canada. He has held academic positions at Carleton University, Ottawa, and the University of Canterbury, Christchurch, New Zealand. He joined the University of Victoria, in 1999, and is currently a Professor with the Department of Electrical and Computer Engineering. He became a Fellow of the Engineering Institute of Canada in 2002 and a Fellow of the Canadian Academy of Engineering

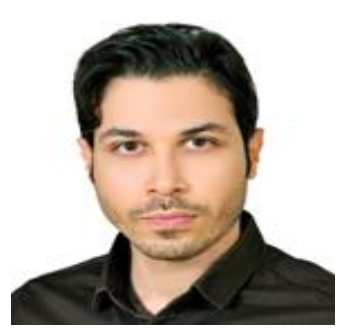
in 2012. His research interests include information theory and communication theory, algebraic coding theory, cryptography, multicarrier systems, smart grid, intelligent networks, cryptography, and security. 